\title{
Akif Pirinçci „Yin“ - Geschlechterforschung oder groteske Dystopie
}

\author{
Gülrû Bayraktar (D), Alanya
}

https://doi.org/10.37583/diyalog.1030292

\begin{abstract}
Deutsch)
Akif Pirinçci ist einer der meist diskutierten Autoren der neueren deutschen Literatur. Dies liegt nicht nur an seiner Arbeit als Schriftsteller, sondern auch an seinen provokativen Aussagen. Immer wieder taucht sein Name in Presseberichten auf, die im Zusammenhang mit Frauenfeindlichkeit oder sogar Rassismus stehen. Diese Schlagwörter ziehen sich auch durch seine Romane. Das wiederum wirft die Frage auf, ob Akif Pirinçci geschlechtsspezifische, negative Ideologien in seinen Werken versucht zu suggerieren. Der Roman „Yin“ stellt eine neue Weltordnung dar, in der es keine Männer mehr gibt und die Frauen auf sich selbst gestellt sind. Die Art und Weise in der das Thema von Pirinçci behandelt wird, ist teilweise so grotesk, dass die Intention des Autors irritierend auf den Leser wirken kann. Diese Arbeit analysiert werkimmanent den Roman „Yin“ von Akif Pirinçci hinsichtlich der Fragestellung, ob der Autor mit dem Roman versucht einen Beitrag zur Geschlechterforschung zu liefern, oder ob es sich um eine reine Dystopie handelt.
\end{abstract}

Schlüsselwörter: Geschlechterforschung, Dystopie, grotesk, Akif Pirinçci, Yin.

\section{Abstract (English)}

Akif Pirinçci „Yin“ - Gender studies or grotesque Dystopie

Akif Pirinçci is one of the most discussed authors in recent German literature. This is not only due to his work as a writer, but also to his provocative statements. More than often his name appears in press reports related to misogyny or even racism. These slogans also run through his novels. This in turn raises the question of whether Akif Pirinçci tries to suggest gender-specific, negative ideologies in his works. The novel "Yin" represents a new world order in which there are no more men and women are left to fend for themselves. The way in which the topic is dealt with by Pirinçci is sometimes so grotesque that the author's intention can be irritating to the reader. This work inherently analyses the novel "Yin" by Akif Pirinçci with regard to the question of whether the author tries to contribute to gender studies with the novel or whether it is a pure dystopia.

Keywords: Gender Studies, Dystopie, grotesque, Akif Pirinçci, Yin. 


\section{EXTENDED ABSTRACT}

Literature is always a mirror of society. It contains so many facets of cultural assets that the reader can see himself and society in this mirror. Authors often try to draw attention to a very specific problem in society with their novels. The author's attitude and his way of looking at things play an important role. If it is the intention of the author to bring a certain thing into the focus of the reader, then the result is often a subjective literature. Throughout human history, the society has often witnessed how texts can change people's minds and even direct them in a deliberate negative direction. Often the unconscious reader has no chance to protect himself from agreeing to a certain opinion. This can lead to ideas, ideologies, stereotypes which are being implicated to the reader. Opinions are formed unconsciously and are unfortunately recognized much too late. The reader gets a distorted picture of reality and only becomes aware of it, if at all, when he or his environment have been damaged by it. With the help of literature analyses, such texts can be clearly examined. This is the only way to protect the reader from accepting unconscious suggestions. The reader has the chance to understand the read text through a neutral view.

In this case, too, the aim is to give the reader the chance to gain awareness according to the novel "Yin" by Akif Pirinçci. Akif Pirinçci is one of the most discussed authors in recent German literature. This is not only due to his work as a writer, but also to his provocative statements. More than often his name appears in press reports related to misogyny or even racism. So it seems like, no matter in which absurd way, he just wants to be noticed. He parodies foreigners in various public videos and thereby ensures his constant presence in the press. Sexist, racist or even divisive statements are part of his language for him. These slogans also run through his novels. This split can be found in almost all of his novels. Be it the division of the races in his seemingly naive cat novels or the division of the sexes in his Apocalyptic works. This raises the question of whether Akif Pirinçci tries to suggest gender-specific, negative ideologies in his works.

Pirinçci is an exceptional author for sure, which is not only reflected in his choice of subjects but also in his talent to play with words and symbols in his novels. Without question, he has the gift of juggling with words. He knows how to get the reader carried away. His novels immediately transport the reader into an often absurd but understandable world. This of course makes the author as a strategist all the more dangerous, because the intention behind the texts, if there is one, could lead the reader being wrongly oriented.

The novel "Yin" represents a new world order in which there are no more men and women left to fend for themselves. The way in which the topic is dealt with by Pirinçci is sometimes so grotesque that the author's intention can be irritating to the reader. This work inherently analyses the novel "Yin" by Akif Pirinçci with regard to the question of whether the author tries to contribute to gender studies with the novel or whether it is a pure dystopia.

The illustrated basic elements of dystopia make it clear that Pirinçci consciously opted for a dystopia. It is not his aim to present or propose a new, better world order. He is not in the least interested in contributing to the gender studies. His focus is on dystopia to show that Eve cannot be without Adam. If this were not his main intention, he would have created a Utopia. A land of milk and honey in which women get along very well, if not better, even without men.

This leads to the conclusion, also based on the analysis results, that Akif Pirinçci makes no contribution to gender studies with his novel "Yin", despite the scientific arguments presented in this way. The novel is exclusively a dystopia and should be understood as such. 


\section{Einleitung}

Die deutsche Literatur hat über die Geschichte hindurch viele verschiedene Entwicklungen erfahren. Angefangen von der mündlich tradierten Literatur bis hin zur Gegenwartsliteratur hat sie sowohl durch innere, als auch durch äußere Einflüsse verschiedenste Facetten erhalten. Eine dieser Facetten ist die Migrationsliteratur, die von den Autoren ins Leben gerufen wurden, die entweder von Gastarbeiterfamilien abstammen oder selber nach Deutschland ausgewandert sind. Autoren dieses Genres waren lange Zeit der Diskussion ausgesetzt, was denn eigentlich die Migrationsliteratur und Autoren dieser Literatur ausmacht. Innerhalb dieser Phase haben sich viele Autoren von diesem Begriff absichtlich distanziert.

Autoren, die sich nicht mehr mit ihrer Abstammung, sondern mit ihrer neuen Heimat und der hiesigen Identität identifizierten, zeigten sich ehrgeizig darin zu beweisen, dass sie Autoren waren, die so weitgefächert schreiben konnten, dass sie nicht in die Schublade der Migrationsliteratur hineinpassten. Einer dieser Autoren ist Akif Pirinçci. Sein Wegstreben vom, deutschsprachigen Autor ${ }^{6}$ und sein hinstreben nach Anerkennung als , deutscher Autor' spiegelten sich nicht nur in seinen Romanen wieder, sondern auch in seiner Haltung im öffentlichen Leben. In seinen Interviews deklariert er immer wieder ein deutscher zu sein.

Ich würde mich durchaus als Deutschen bezeichnen. Ich bin, glaube ich, sogar ein bisschen Deutscher als manch ein Deutscher. (Heinrich 2014a: 2'03")

Doch im Laufe der Zeit, als der Autor seine totale finanzielle und somit auch gesellschaftliche Unabhängigkeit erlangt, hört und liest man immer mehr von Skandalen Pirinçcis. Sein sogenanntes ,Deutsch sein' verfällt in einen eher rassistischen, intoleranten Ton, so dass er sich in Interviews, Presseerklärungen oder auch auf Lesungen gegen den Islam, gegen Ausländer und auch Homosexuelle äußert (Schulze 2015: o. S.). Auch sein Bild von der Frau in der Gesellschaft wird in den Medien oft kritisiert (Lehmann 2015: o.S.). Doch was steckt hinter diesem Skandalmacher und worin liegt eigentlich sein literarisches Streben? In dieser Arbeit wird versucht im Roman „Yin“von Akif Pirinçci, anhand der werkimmanenten Literaturanalyse zu erörtern, ob es Ziel des Autors ist eine Art Geschlechterkampf über die Literatur auszufechten, oder ob sich sein Roman um ein Hiobsszenario, um eine Dystopie handelt.

\section{Die Person Akif Pirinçci - Kein Migrationsliterat per se}

Von Akif Pirinçci als Migrationsliterat zu sprechen, wäre wohl kaum in seinem eigenen Sinne, noch käme es der Definition des Begriffs nahe. Es ist nicht zu leugnen, dass er als Kind einer Gastarbeiterfamilie nach Deutschland kam. Doch sein persönlicher und literarischer Werdegang sprengen tatsächlich alle Ufer dieses Begriffs. In diversen Interviews unterstreicht er, dass er nicht als „Autor der Migrationsliteratur“ abgestempelt werden will. In der Diplomarbeit von Ana Andric, kommt die Frage auf, ob es sich bei allen nicht deutschstämmigen Autoren um Migrationsliteraten handelt: ,, Nell fragt sich zum Beispiel, ob man den in Deutschland lebenden und aus der Türkei stammenden Autor 
Akif Pirinçci zu „,Migrantenliteratur “ zählen kann, obwohl Migration nur eine marginale Rolle in seinen Texten spielt" (Andrić 2012: 33).

Schon in jungen Jahren hat er eine starke Neigung zu schreiben. Trotz seiner eher bescheidenen Schulausbildung, ist Schreiben scheinbar sein Lebensmittelpunkt. Als knapp zwanzig Jähriger ist sein erster Roman Tränen sind immer das Ende geschaffen. Schon hier wird man eher aufgrund seiner provokativen Haltung auf Pirinçci aufmerksam. Er sieht sich selbst als reiner Deutscher. Für ihn ist die Integrationsdebatte um die Generation der Gastarbeiterkinder eine rein Fiktive. Ganz anders als die bislang bekannten Autoren der Migrationsliteratur äußert Pirinçci, dass es eigentlich keine Identitätsdiskrepanzen geben kann.

Die fühlen sich auch gar nicht zerrissen, so etwas gibt es in Wirklichkeit gar nicht. Das reden sie den Deutschen nur ein. Damit sie von hinten bis vorne bedient werden. [...] Das sind alles eigentlich Quatsch-Behauptungen von Soziologen, dass sie sich innerlich zerrissen fühlen, dass wenn sie durch die Straßen gehen, sich fragen würden bin ich ein Türke, ein Araber oder ein Deutscher oder sowas. (Conrad 2014: 3'32")

Als Akif Pirinçci zum Ende der 80er Jahre mit seinem Roman Felidae bekannt wurde, schien sich sein Wunsch sich als ,echter deutscher Autor ${ }^{6}$ zu etablieren, verwirklicht. Felidae ist eine ,Fabelhafte' Geschichte einer Sherlock Holmes Katze und ein in sich hervorragend zusammengestellter Krimi. Bereits in diesem Roman lassen sich Züge Pirinçcis nachvollziehen, die sich als bizarr oder gar grotesk definieren lassen. In den vorgeschlagenen Definitionen von Grotesk sind wir häufig mit zwei gegensätzlichen Haltungen konfrontiert: Grotesk ist für die einen ein Genre und für die anderen ein Stil (Aktulum 2020: 6). Doch die Darstellung der ermordeten Katzen ist so präzise, so extravagant und eindeutig in jeder detaillierten Abscheulichkeit, dass der Leser direkt ein Kopf-Kino abspielt. Das macht Pirinçci zu einem so machtvollen Autor, der das Groteske als Stil gebraucht. Man möchte die Hand vor Augen halten und doch weiterlesen.

Wie gesagt, ich komme von der Schriftstellerei. Ich will Emotionalisieren. Ich habe in meinen Romanen, weiß nicht, bestimmt schon zehntausend Menschen umgebracht. Einmal habe ich sogar beinahe die Atombombe gezündet und die Welt vernichtet. Wie gesagt, ich möchte Emotionalisieren. (Grell 2016: 42'16")

Bei Felidae ist diese bizarre Eigenart als Autor noch eingehüllt in den flauschigen Pelz der zahlreichen Katzen des Romans. Später als in Pirinçcis Romanen der Übergang zum Menschen geschieht, verfällt jegliche Art des Wohlgefühls und es bleibt die nackte Darstellung des Grauens.

In der Recherche um Pirinçci lässt sich schnell feststellen, dass Akif Pirinçci vom Schreiben lebt. Nicht nur im Sinne des Einkommens, sondern er scheint durch das Schreiben zu atmen, zu leben. Auf seiner Webseite sind unzählige Artikel zu finden, wie sie kaum bei einem anderen Autor zu lesen sind. Jegliches Gefühl, jegliche Anschauung, jede Empörung muss transkribiert werden. Dies scheint seine Superpower zu sein, oder sein Auslassventil, oder auch sein Fluch. Denn jegliche Schreibarbeit, die Pirinçci nach seinen Katzen-Romanen geleistet hat, sorgt gesellschaftlich, wie auch politisch für Furore. In diversen Schlagzeilen renommierter Zeitungen heißt es;

Akif Pirinçci sieht seine Existenz zerstört. (Die Welt 2015: o. S.) 
Lutz Bachmann entschuldigt sich für Pirinçci-Auftritt. (Süddeutsche Zeitung 2015: o.S.)

Pirinçci ist in viele verschiedene Diskussionen verwickelt. Seine Kundgebungen zu Religionen, zu Rassen und auch zu Frauen bringen ihn immer wieder in diverse Schlagzeilen. Schon die Titel seiner neuesten Arbeiten sprechen für sich; Deutschland von Sinnen-Der irre Kult um Frauen, Homosexuelle und Zuwanderer, Das Schlachten hat begonnen und Umvolkung sorgen für heftige Debatten. Alle diese Bücher scheinen aus einer Wut heraus entstanden zu sein. Pirinçci versucht aus seiner Sicht darzustellen, dass er sich das alte Deutschland zurückwünscht und was es braucht um dorthin zurückzukommen.

Ich möchte mein altes Deutschland wiederhaben. Es war so toll. (Conrad 2014: 6'54")

In seinem Roman „Yin“ fokussiert er sich auf die Frauen. Viele Kritiker stellen die Frage, ob es sich bei diesem Buch um „einen Rundumschlag gegen die Frauenwelt handele?“ Doch Pirinçci schreibt im Anhang seines Buches eine Art Versöhnungsschrift;

Dies ist ein Roman und keineswegs die Abrechnung des Autors mit ,der anderen Hälfte des Himmels', unterstützt durch gezielt ausgesuchte, manipulierte Fakten. (Pirinçci 1997: 825)

Um jedoch zu verstehen, ob es sich bei dieser Äußerung nicht doch um eine Ironie des Autors handelt, muss der Text näher analysiert werden.

\section{„YIN“ - Eine Welt ohne Männer}

In seinem Roman „Yin“ lassen sich bereits die Vorstufen der oben dargestellten skandalträchtigen Gefühlsaufruhr Pirinçcis erkennen. Das Thema Frauen und Männer im Vergleich wird in seinem Roman gründlich erörtert. Akif Pirinçci erklärt in seinem Interview mit Thorsten Heinrich, dass ihn Themen rund um Genetik, Evolution und Geschlechterunterschiede faszinieren und dass er schon ein Profi auf diesem Gebiet sei (Heinrich 2014: 1'14'37").

Pirinçci beginnt sein Buch direkt Provokativ mit einem Vers aus der Genesis, in dem es in Bezug auf den Sündenfall heißt:

Nach deinem Mann wird dein Verlangen sein, er aber wird über dich herrschen.

Genesis/ Der Sündenfall (Pirinçci 1997: 5)

Dies passt zu seiner Überzeugung, dass die Herrschaft des Mannes über die Frau eine Art Erfindung sei.

Frauen werden durch die Bank von Männern unterdrückt. Das ist einfach eine Erfindung, einfach eine blöde Erfindung. (Heinrich 2014b: 7'58")

Direkt auf der darauffolgenden Seite ist ein Absatz zu lesen, in dem Pirinçci darüber berichtet, dass der Biologe David Crews eine in Texas lebende Eidechse beobachtet hat, von der es keine Männchen gibt. Die Weibchen sind in der Lage sich selbst zu reproduzieren. Dieser Absatz scheint den Denkanstoß für das Konzept des Buches gegeben zu haben. 
Noch bevor es zu den Frauen im Roman kommt, legt Pirinçci noch eine Provokation drauf und zitiert aus Rufus Salamander Text „Wie man Frauen wirklich kriegt und sie sich untertänig macht“. Der Text beginnt mit dem Namen „ADAM - Das Herz des Mannes“. Hier wird umfangreich erläutert, dass ,,das Herz des Mannes ein ,verwunschener Ort ist, von dem niemand weiß, was darin vorgeht" (Pirinçci 1997: 7).

Dass Pirinçci den Mann und die Beschreibung des männlichen Herzens voranschickt, lässt schon erahnen, dass es in dem Roman darum geht, wie unterschiedlich das männliche und das weibliche Herz ticken.

Auch der Name des Romans „Yin“ scheint aus dem Gedanken der fernöstlichen Philosophie speziell vom Daoismus herzurühren. Yin und Yan symbolisieren die sich ergänzenden, einander entgegengesetzten aber trotzdem aufeinander bezogenen kosmischen Prinzipien. Yang wird definiert als hell, hoch, hart, heiß, positiv, aktiv, bewegt, männlich und das schwarze Yin dunkel, weich, feucht, kalt, negativ, passiv, ruhig, weiblich (van Ess 2011: 16). Jede Seite besitzt sein Pendant im anderen, was auch dem Aufbau der Geschichte von Pirinçci gleichkommt.

Im Roman selbst werden sieben Frauenschicksale dargestellt. Die Besonderheit dieser Frauen ist, dass sie sich alle in einer Zeit befinden, in der ein Virus die Männer langsam vernichtet hat. Der Virus hat es auf das Y- Chromosom abgesehen. Deswegen überleben alle Frauen verlieren aber ihre Väter, Partner und Söhne. Die Frauen sind nun auf sich selbst gestellt und müssen in einer neuen Weltordnung zurechtkommen. Doch zu Beginn sind sie so stark von ihrer Trauer, übermannt', dass in der Zeit bis sie sich aufgerafft haben, viele Errungenschaften der Welt zugrunde gehen. Von der Rohstoffversorgung bis $\mathrm{zu}$ verschiedensten Industriezweigen geht alles unter. Ein mittelalterlicher Zustand wird dargestellt, eingebettet in der Kulisse der Neuzeit. Über Szenen aus der Vergangenheit zeigt uns Pirinçci das Leben der Frauen vor der Teilapokalypse und stellt gegenüber wie die jeweilige Frau mit der jetzigen Situation fertig wird. Die Rückblenden sind durchwoben von Folter, Perversionen, Hass und Erotik. Pirinçci vermengt abstoßende Szenen mit einem gewissen Humor, der es dem Leser erleichtert den Stoff zu schlucken.

Er sieht aus wie Joop. [...]. Joop Jesus, errette mich! (Pirinçci 1997: 254)

Jede der dargestellten Frauen ist zwar in ihrer Person einzigartig, repräsentiert jedoch auf eine gewissen Weise bestimmte Frauen dieser Welt. Da gibt es die Frau, deren Schönheit zu ihrem Laster wird. Die Maskuline, die Zerbrechliche, die durch Liebe zur Prostitution Gezwungene, die Hässliche, die Mutter usw. Auf einer bestimmten Ebene verschmelzen die Einzelschicksale miteinander.

Die erste im Roman von Pirinçci dargestellte Frau ist die ehemalige Journalistin Helena. Schon die Wahl ihres Namens verrät viel über ihre Weiblichkeit und ihre Geschichte. Ihr Kapitel beginnt mit der Einleitung - Die Erinnerung. Wie allen Frauen im Roman ist auch ihr eine männliche Figur gegenübergestellt. In diesem Fall ist es ihr Vater. Es handelt sich hierbei um einen Vater, der ihre Mutter so unglücklich gemacht hat, dass Helena sich ausschließlich an das Weinen ihrer Mutter erinnert. Er ist ein notorischer Betrüger und hat unzählige Verhältnisse mit anderen Frauen. So schmerzlich 
diese Situation auch für Helena ist, kann sie sich nicht vor der Liebe zu ihrem Vater schützen.

Und trotzdem... Trotzdem hatte sie ihn geliebt, so sehr geliebt, dass sie sogar zum Inzest bereit gewesen wäre. (Pirinçci 1997: 12)

Helenas Vater repräsentiert förmlich alle Männer, die das Vertrauen der Frauen in die Männer schon in der Kindheit vernichten. Alle weiteren Beziehungen vollziehen sich somit schon unter diesem Zeichen. Nach dem alle Männer ausgestorben sind, leben Helena und ihr Pferd Pegasus ganz alleine ohne Kontakt zu anderen Menschen. Pirinçci beschreibt Helenas Beziehung zu ihrem Pferd auf eine etwas außergewöhnliche Weise. In einer Szene, in der Helena nackt in einen See steigt, ihr Pferd ihr folgt und sie sich dort nahekommen, ist eine starke Erotisierung feststellbar. Der Leser erhält schnell das Gefühl in einer Geschichte aus der griechischen Mythologie gelandet zu sein, in der eine Amazone mit ihrem Pferd badet.

Und aus diesem Na-warte wurde der Beginn einer Affäre, auf die selbst Helden und Heldinnen italienischer Fotoromanzen neidisch gewesen wären. Leidenschaft, wie sie sie Menschen gegenüber niemals empfunden hatte, und Leidenschaft, die er sich trotz der erlittenen Hölle bewahrt hatte wie eine Vulkanlandschaft, eine trotzig emporsprießende Blume. (Pirinçci 1997: 34)

Die zweite von Pirinçci vorgestellte Frau ist Margrit. Sie ist schon beinahe als Hauptprotagonistin einzustufen, da sie die Hauptdynamik der Ereignisse entfacht. Sie wird als unattraktive, penible, Reinheit liebende, ordentliche, übergroße, starke Frau dargestellt.

Wenn alles vor Sauberkeit glänzte und alle anstehenden Aufgaben erledigt waren, hatte sie das Gefühl, dass sie ein ins Chaos abgleitendes Universum im letzten Augenblick gerettet hatte. (Pirinçci 1997: 43)

Die männliche Komponente zu Magrit ist ihr Mann, den sie direkt zu Beginn des Kapitels eigenhändig ins Grab legt. Es geht bei dem ihr gegenübergestellten Mann nicht um seine Person an sich, sondern viel mehr um die Wirkung, die Margit auf Männer hat. Pirinçci nimmt hier kein Blatt vor den Mund und schreibt: „, Sie war hässlich, war schon immer hässlich gewesen. In anderen Kreisen hätte man wohl unattraktiv gesagt, was aber, wie sie fand, auf dasselbe hinauslief" (Pirinçci 1997: 40).

Um noch genau zu verdeutlichen, was genau Margit so hässlich macht, ergänzt Pirinçci:

Margit war achtundvierzig Jahre alt, maß einhundertneunzig Zentimeter und wog Hundertzwanzig Kilo. (Pirinçci 1997: 40)

Doch Magrit, mit ihrer obsessiven Ordnungsliebe, erkennt schnell die Chancen in der neuen Weltordnung. In den Rückblicken auf ihre Vergangenheit zeigt Pirinçci, dass Margit schon immer berechnend und zielstrebig war. Auch heute kann sie nichts von ihrer neuen Besessenheit abbringen. Sie versteht sofort, dass das wertvollste auf der neuen Welt die Samenbanken sind. Durch die Kontrolle der Samenbanken erhofft sie sich Weltmacht. 
In einer Männerlosenwelt kam diesem weißen Gel eine größere Bedeutung zu als Geld,

Edelmetallen, Uran oder menschlicher Intelligenz, ja, selbst einem Menschenleben.

(Pirinçci 1997: 89)

Die dritte im Bunde ist Lilith. Auch hier ist Name Programm. Die schöne Lilith verliebt sich in einen Mann, der sie nach einiger Zeit dazu treibt sich für ihn, oder besser für seine angeblichen Schulden zu prostituieren. Dieser Zustand führt nach langer Zeit in diesem Milieu dazu, dass Lilith zu einer Person mutiert, die nicht mehr fähig ist jemanden zu lieben. Verglichen mit den anderen in diesem Buch erwähnten Frauen, schildert Pirinçci Liliths Leben mit allen Facetten und Einzelheiten. Der Leser bekommt Dekaden ihres Lebens vorgeführt. Der Autor lässt anhand der dargestellten Prostituierten und Freiern seiner ganzen Phantasie freien Lauf. Dem Leser bleibt keine Perversion erspart, aber genau dadurch wirkt die Geschichte so real. Als es mit den Männern zu Ende geht, scheint Liliths Schicksal doch eine Wende zu nehmen. Einer ihrer langjährigen Freier, der seine letzten Minuten in der Gesellschaft von Liliths Sinnlichkeit verbringen will, hinterlässt ihr Goldbarren und diverse Luxusgüter.

Für dreißig Goldbarren kannst du dir sogar eine Polaroid davon schießen und es an deinen Sargdeckel pinnen, lieber Oscar, huschte es ihr beschwingt durch den Kopf. Sie deponierte seinen Kopf behutsam auf die Sofalehne, stand auf und stellte sich breitbeinig vor ihm hin. (Pirinçci 1997: 200)

Akif Pirinçci erzählt an vierter Stelle von Viola. Viola ist ein ehemaliges, internationales Top-Model. Doch von ihrer einstigen Schönheit ist nichts mehr übrig. Das liegt an der Gestalt, die Pirinçci als Pendant an Violas Seite stellt. Es ist ihr Peiniger, ihr Folterer. Ein Psychopath, der Viola entführt und über etwa zwei Jahre in Gefangenschaft auf das äußerste Quält. Schlimmer noch als die Begebenheiten bei Lilith stellt Pirinçci die zwei dunklen Jahre von Viola dar. Von unzähligen Vergewaltigungen auf die schlimmste Art und Weise bis hin zur Zwangsabtreibung muss sie unglaubliche Qualen erleiden. Auch hier bleibt dem Leser nichts erspart. Pirinçci führt seine ganzen Künste vor.

Sie schrie. Es war der Schrei der Wahnsinnigen, schrill, auf eine bizarre Art melodiös, wie die unergründliche Weise eines berauschten Eingeborenen, der die Geister heraufbeschwört. (Pirinçci 1997: 256)

Als am Ende der Seuche ihr Peiniger sich dem Schicksal aller anderen Männer anschließt, ist Violas Körper so entstellt, dass sie nicht wiederzuerkennen ist. Margit findet sie und macht sie zu ihrer Verbündeten. Inzwischen ist Viola durch ihre Pein zu einem Unmenschen mutiert und somit genau die richtige Komplizin für Margit und ihren Wunsch nach Weltmacht.

An nächster Stelle wird Vanessa vorgestellt. Vanessas Leben wird von ihrer Karriere bestimmt. Sie ist Politikerin und das männliche in ihrer Geschichte ist eigentlich das ganze Patriarchat. Vanessa verachtet das von Männern errichtete System. Doch ebenso sehr verachtet sie auch die Frauen. Besser gesagt, sie fand alle Frauen zu Dumm für diese von Männern strukturierte Welt.

Zwar fanden Männer von ihren entlarvungssüchtigen Röntgenblicken auch keine Gnade, doch glaubte sie, dass Dummheit des Weibes bei weitem alles übertraf, was die Palette des menschlichen Fehlverhaltens zu bieten hatte. (Pirinçci 1997: 349) 
Vanessas Aufgabe besteht in dem Buch darin, dass sie jetzt als freie Politikerin, erlöst von der Männerwelt, aber unter den Fittichen von Margit, bestimmen kann, wer von nun an Kinder bekommen darf und wer nicht.

In diesem Zug kommt auch schon die nächste Figur ins Licht der Darstellung. Es handelt sich um Angelika. Sie ist die Ärztin in der Gruppe. Die Gentechnik ist ihre Stärke und der Mann an ihrer Seite ist Hagen. Er wird als tölpelhafter Typ dargestellt, der äußerlich zwar einem germanischen Gott nahekommt, geistig mit Angelika aber nicht mithalten kann. Angelika hat einen starken Kinderwunsch. Es stellt sich leider raus, dass sie aufgrund ihrer traumatischen Vergangenheit, nicht mehr im Stande ist Kinder zu bekommen. Das treibt sie dazu an, sich noch tiefer in die Materie der Genforschung einzuarbeiten. Mit der neuen Weltordnung erhält sie die Chance ihren eigenen Kinderwunsch bei anderen Frauen zu verwirklichen.

Vergangen war bald der eigene Kinderwunsch, und der unerfüllte Kinderwunsch wurde für sie zum Mittelpunkt ihres Lebens. (Pirinçci 1997: 553)

Auch der Ort, an dem Angelika arbeitet spielt in dem Roman eine wichtige Rolle. Angelika arbeitet in dem größten Genlabor der Welt. Innerhalb dieses Genlabors befindet sich eine enorme Samenbank. Im weiteren Verlauf des Romans verbündet sich Angelika mit Margit, um ihr gemeinsames Vorhaben zu optimieren.

Als letztes wird dem Leser noch Cora vorgestellt. Sie ist ganz anders als alle anderen Frauen. Sie scheint keine vergleichbaren Traumata in ihrer Vergangenheit erlebt zu haben. Ihre Leidensgeschichte beginnt mit dem Aussterben der Männer. Sie ist Pilotin und als Margit davon erfährt, entführt sie Coras Töchter. Margit zwingt damit Cora für sie zu arbeiten. Ihre Aufgabe soll es sein, alle Samenbanken dieser Welt aus der Luft zu bombardieren. Cora ist gezwungen zu kapitulieren. Doch als Margit nach 12 Jahren Coras Töchter immer noch nicht freigibt, übt Cora Rache.

Während Margit, Viola, Angelika und Vanessa sich im Laufe der Geschichte verbünden, um die Weltmacht an sich zu reißen, werden Helena und Lilith ausgegrenzt. Später entwickeln sich aus diesen beiden Gruppen, bei der Cora notgedrungen zu Margit übergeht, zwei Fronten, die sich auf das äußerste bekriegen. Es beginnt ein Krieg zwischen den Frauen, die für die künstliche Befruchtung sind und den Frauen, die sich für die natürliche Befruchtung einsetzten.

Mit ,Adam - Das Herz des Mannes` begonnen, beendet Pirinçci sein Buch mit dem letzten Kapitel ,Eva - Das Herz der Frau‘. Auch hier zitiert er Rufus Salamander und schließt somit den Kreis.

\section{Pirinçci, Geschlechterforschung und Dystopie}

In Anbetracht dieses Handlungsstranges sollte zunächst eine genaue Begriffsbestimmung vollzogen werden. Wenn in der Literatur der Begriff Geschlechterforschung gebraucht wird, dann ist die Rede von literarischem Material, dass eine gewisse geschlechterspezifische Problematik behandelt. 
Geschlechterforschung fragt nach der Rolle von Geschlecht bei der Verteilung von politischer, sozialer und ökonomischer Macht und Ressourcen in unterschiedlichen Bereichen und analysiert Mechanismen und Entwicklungen, welche zu Geschlechterungleichheiten geführt haben. (Georg-August-Universität 2021)

Genderstudies oder auch Geschlechterforschung steht in direktem Zusammenhang mit feministischen Ansätzen. Die Abgrenzung von Frauenforschung und Geschlechterforschung ist noch nicht wirklich eindeutig. Eindeutig ist hingegen, dass es mehrheitlich Frauen sind, die diese Forschung vorangetrieben haben. Eine der wichtigsten Vertreterinnen ist Margaret Atwood. Kuznicki beschreibt ihre Definition von Dystopie wie folgt: „, Definitely, one of Atwood's most powerful means of expressing worldly interests-because it seems that, after all, substance always comes before form in her prose - is the genre of dystopian or speculative fiction, as both terms are adequate" (Kuźnicki 2017: 15). Wenn es um Forschungsinhalte wie kulturelle Geschlechtszuweisung, Machtverhältnisse, soziale Ungleichheit, soziale Stellung oder auch geschlechtsspezifische Bildung geht, so waren es bislang eher Frauen, die dieser Forschung nachgegangen sind (Steffen / Rosenthal / Vaeth 2004: 9).

Außerdem sind es, ganzgleich wie kritisch der Diskurs auch ist, stets Forschungen, nach der Verbesserung der Situation der Frau innerhalb der Gesellschaft. Dies lässt sich vor allem in der Arbeit von Judith Butler erkennen, die als Vorreiterin der Queer-Theorie die Aspekte der Kulturen in Bezug auf Geschlechterrollen und der sich daraus entwickelnden Ausbeutungsverhältnisse erforscht.

\section{Die kulturellen Konfigurationen von Geschlecht und Geschlechtsidentität könnten sich vermehren, [...] indem man die Geschlechter-Binarität in Verwirrung bringt und ihre grundlegende Unnatürlichkeit enthüllt. (Butler 1991: 218)}

Es geht also in der Geschlechterforschung nur darum, die Situation der Geschlechter deskriptiv darzulegen, sondern es besteht die Bemühung eine Verbesserung im Miteinander zu schaffen. Genau dieser Aspekt fehlt in dem Roman von Akif Pirinçci. Pirinçci stellt sieben verschiedene Frauenschicksale dar. Jede von ihnen hat das Potenzial, Gegenstand einer umfangreichen Studie zu werden. Er stellt alle Facetten der Gesellschaft vor. Die reiche Frau, die arme Frau, die schöne Frau, die hässliche Frau und so weiter. Doch findet sich in dem Roman in keiner Zeile ein Vorschlag, um diese Frauen aus ihrer Misere zu befreien. Genau deshalb lässt sich der Roman nicht eindeutig der Geschlechterforschung zuordnen.

Auf der anderen Seite haben alle Frauen im Buch von Akif Pirinçci eines gemeinsam. Sie haben das Problem Mann. Dieses Problem wird vom Autor so explizit dargestellt, dass an dieser Stelle die Brücke zu der Geschlechterforschung geschlagen werden könnte. Denn sei es das Körperkonzept, das Geschlechterverhältnis oder die politische Problematik, Pirinçci zeigt sie allesamt auf. Die Lösung, die Pirinçci für das gemeinsame Problem dieser Frauen bietet; er lässt alle Männer aussterben. Da dies definitiv keine Lösung im Rahmen der Geschlechterforschung ist, liegt bei weiterer Überlegung auf der Hand, dass hier das Rad zur Dystopie geschlagen werden kann. Denn eine Dystopie oder auch Anti-Utopie ist laut der Definition von Wilpert ein in den meisten Fällen in der Zukunft spielendes Szenario, ,[...] nicht im imaginierten Nirgendwo, 
sondern in e. anderen, gleichzeitigen, fernen [... $]^{\text {" }}$ in der unerwünschte oder sogar katastrophale Gesellschaftsordnungen dargelegt werden (Wilpert 2001: 865).

Ausgehend von dieser Definition lassen sich die Strukturen des Romans nach diesen Merkmalen analysieren. In der Literaturanalyse einer Dystopie sind drei Grundelemente vorhanden:

1-Die Exposition: Das ist die Darstellung aller wichtigen Bereiche der im Roman entworfenen dystopischen Wirklichkeit. Über den oder die Protagonisten werden meist durch schmerzliche Erfahrungen die ungerechte neue Weltordnung greifbar gemacht.

2-Die Entscheidung: Der Protagonist trifft eine Entscheidung, in der sie oder er gegen die vorhandene negative Ordnung aufständisch wird. In der Regel richtet sich diese Rebellion gegen ein Regime oder einen despotischen Staat. Ziel ist es den unerträglichen vorhandenen Zustand aufzuheben.

3-Die Erkenntnis: Im Gegensatz zur Utopie enden fast alle Dystopien damit, dass der Protagonist erkennen muss, dass er oder sie alleine keine Chance gegen den Despoten hat. Der Protagonist wird entweder psychisch in das System zwangsintegriert oder physisch vernichtet (Teoman 2012: 5).

Akif Pirinçci weiß diese Grundelemente in „Yin“ geschickt einzusetzen. Bereits im ersten Kapitel wird mit der Exposition begonnen. Pirinçci beschreibt eine neue Welt, in der es keine Männer mehr gibt. Dies deklariert er nicht direkt im ersten Schlag, sondern verweist auf Einzelschicksale. Er stellt Männer dar, die an ihrem eigenen erbrochenen Ersticken, vereitert in irgendwelchen Straßenecken liegen oder von ihren Frauen beerdigt werden.

Sie legte die Leiche auf dem Rasen ab und begann eine Grube auszuheben. Noch vor drei
Jahren hätte die Presse eine Frau, die im Vorgarten ein Grab für ihren Ehemann schaufelt,
garantiert mit Schlagzeilen gewürdigt - und die Polizei mit einer
Wachsfigurennachbildung im Kriminalmuseum. (Pirinçci 1997: 97)

Somit besteht die Gesellschaft in der von Pirinçci gestalteten neuen Welt ausschließlich von Frauen. Auch wenn es nun nicht die Hauptprotagonistin gibt, erschafft Pirinçci doch ein Cluster von Frauen, die sowohl die Protagonisten, als auch die Einzelschicksale darstellen, die es für eine Dystopie bedarf.

Die Bedingung des zweiten Grundelements einer Dystopie wird von Pirinçci auch erfüllt. Die Protagonistin oder Protagonistinnen treffen eine Entscheidung. Hier spaltet Pirinçci sein Cluster in zwei Fraktionen. Dies hält die Dynamik von Gut und Böse aufrecht. Die eine Gruppe von Frauen entscheidet sich die neue Weltordnung in die Hand zu nehmen und an die Macht zu kommen. Die zweite Gruppe entscheidet sich dagegen. Somit stellen die Protagonistinnen der ersten Gruppe eigentlich das Regime der Dystopie dar. Die zweite Gruppe von Frauen, die gegen das Vorhaben rebellieren sind somit die Träger des Grundelements der ,Entscheidung'.

Es tut uns leid, wenn wir Sie mit unserem überfallartigen Auftreten erschreckt haben sollten. [...] Doch durch eine Reihe seltsamer Zufälle sind wir zur Ansicht gelangt, dass Sie und die Spermabestände des Instituts sich in akuter Gefahr befinden. Wir sind gekommen, um Sie zu warnen. (Pirinçci 1997: 608) 
Helena und Lilith sind somit in dem Roman die Protagonisten, die sich gegen das von Margit errichtete Regime der Frauen auflehnen, die die Entscheidungsmacht darüber haben, wer denn nun Gebären darf und wer nicht. So sehr die beiden auch versuchen gegen Margit anzugehen, ihre Bemühungen sind vergebens. Somit tritt auch das dritte und letzte Grundelement der literarischen Dystopie in Kraft. Pirinçci macht durch die Erkenntnis der Protagonisten klar, dass die Weltordnung der Frauen zum Scheitern verurteilt ist.

Das Frauenreich der Lebenden hat sich als ein Flop erwiesen, Schätzchen. (Pirinçci 1997: 817)

Die beiden Protagonisten müssen einsehen, dass sie allein, nichts ausrichten können, gegen das neue System. Sie werden von Margit in eine Falle gelockt und fast ermordet.

Die Wahrheit war, dass man sie, Lilith und Cora nur deshalb ins Reich gelockt hatte, um sie plausibel und in aller Eindringlichkeit von einer Sache zu überzeugen: Die Rebellen müssten den Kontinent verlassen. (Pirinçci 1997: 801)

\section{Schlussfolgerung}

Wie durch die dargestellten Grundelemente der Dystopie deutlich wird, entscheidet sich Pirinçci bewusst für eine Dystopie. Es liegt nicht in seinem Sinne eine neue, bessere Weltordnung darzustellen oder vorzuschlagen. Es geht ihm nicht im Geringsten darum einen Beitrag zu der Geschlechterforschung zu liefern. Sein Augenmerk gilt der Dystopie, um darzustellen, dass Eva nicht ohne Adam kann. Wenn dies nicht seine Hauptintention wäre, so hätte er eine Utopie geschaffen. Ein Schlaraffenland, in der die Frauen auch ohne Männer sehr gut, wenn nicht sogar besser auskommen.

Auch wenn der Autor wiederholt erklärt, dass nicht er es ist, der in seinen Romanen agiert, sondern es ausschließlich die Protagonisten sind, so ist doch trotzdem eine Parallele zu finden zwischen seinen provokativen Äußerungen über Frauen und dem Roman „Yin“. Das lässt, auch an Hand der Analyseergebnisse, schlussfolgern, dass Akif Pirinçci mit seinem Roman „Yin“, trotz seiner so dargestellten wissenschaftlichen Beweisführungen, keinen Beitrag zur Geschlechterforschung liefert. Der Roman kann und sollte in dieser Hinsicht als eine Dystopie verstanden werden.

\section{Literaturverzeichnis}

Aktulum, Kubilay (2020): Sanatsal Bir Biçim Olarak Grotesk Nedir? In: Karamanoğlu Mehmetbey Üniversitesi, Cilt 2(Sayı 1), 1-35.

Andrić, Ana (2012): ,Migrationsliteratur' in Österreich. Eine literatursoziologische Betrachtung der ,Migrationsliteratur ${ }^{\circ}$ in Österreich: Universität Wien. Abgerufen von https://core.ac.uk/download/pdf/11600563.pdf (Letzter Zugriff: 12.04.2021).

Butler, Judith (1991): Gender Trouble. Feminism and the Subversion of Identity - Das Unbehagen der Geschlechter: Frankfurt am Main.

Die Welt (2015): Akif Pirinçci sieht seine Existenz zerstört. Dpa. Abgerufen von https://www.welt.de/politik/deutschland/article147943065/Akif-Pirincci-sieht-seine-Existenz- 
zerstoert.html (Letzter Zugriff: 24.02.2021).

Georg-August-Universitaet (2021): Geschlechterforschung. Abgerufen von https://www.unigoettingen.de/de/638146.html (Letzter Zugriff: 01.03.2021).

Kuźnicki, Slawomir (2017): Margaret Atwood's Dystopian Fiction: Fire Is Being Eaten. Cambridge Scholars Publishing. Lady Stephenson Library: Newcastle upon Tyne, UK.

Lehmann, Armin (2015): Neues vom Hetzer. Der Tagesspiegel. Abgerufen von https://www.tagesspiegel.de/themen/reportage/wer-ist-akif-pirincci-er-redet-gerne-ueber-sexseine-frau-hat-ihn-mit-dem-sohn-verlassen/12484152-2.html (Letzter Zugriff: 14.02.2021).

Pirinçci, Akif (1997): Yin (1. Auflage). Goldmann Verlag: München.

Schulze, Micha (2015): Queer, Die 16 dümmsten und widerlichsten Zitate aus "Die große Verschwulung" 21. Oktober 2015. Abgerufen von https://www.queer.de/detail.php?article_id=24866 (Letzter Zugriff: 13.03.2021)

Steffen, Therese / Rosenthal, Caroline / Vaeth, Anke (2004): Gender Studies: Wissenschaftstheorien und Gesellschaftskritik. Königshausen \& Neumann: Würzburg.

Süddeutsche Zeitung (2015): Bachmann entschuldigt sich für Pirinçci-Auftritt bei Pegida. dpa. https://www.sueddeutsche.de/politik/demonstrationen-bachmann-entschuldigt-sich-fuer-pirincciauftritt-bei-pegida-dpa.urn-newsml-dpa-com-20090101-151020-99-09316 (Letzter Zugriff: 20.02.2021).

Teoman, Zeki Oğuz (2012): Der Endzeitfilm „Dystopie“. University of Applied Sciences, Fakultät Medien: Hochschule Mittweida.

Van Ess, Hans (2011): Der Daoismus: Von Laozi bis heute. Beck C. H.: München.

Wilpert, Gero (2001): Sachwörterbuch der Literatur. Alfred Kröner Verlag: Stuttgart.

\section{Videosequenzen (online)}

Conrad, Susanne (2014): ZDF: Interview mit Akif Pirinçci. https://www.youtube.com/watch?v=6TZcK9ZGFYU (Letzter Zugriff: 22.02.2021).

Grell, Hagen (2016): Doppelstandard der Meinungsfreiheit - Akif Pirincci im NuoViso Talk. https:/gloria.tv/post/2Ktd92A83JA8EEdXfnpEcuxJH (Letzter Zugriff: 04.04.2021).

Heinrich, Thorsten (2014a): Auf eine Zigarre mit Akif Pirincci. https://www.youtube.com/watch?v=6GEQjoDL-1A (Letzter Zugriff: 17.02.2021).

Heinrich, Thorsten (2014b): Auf eine Zigarre mit Akif Pirinçci; 100\% Deutsch und Das Schlachten hat begonnen. https://www.youtube.com/watch?v=v9aiX5POPZg (Letzter Zugriff: 16.02.2021).

Heinrich, Thorsten (2014c): Auf eine Zigarre mit Akif Pirincci (10) - Gender, Frauen, Patriarchat. https://www.youtube.com/watch?v=uFnL1o-_qT0 (Letzter Zugriff: 20.02.201). 